\title{
PSEUDO-HARMONIC MORPHISMS WITH LOW DIMENSIONAL FIBERS
}

\author{
RADU SLOBODEANU
}

\begin{abstract}
Harmonic morphisms with one-dimensional fibers are subject to various classification results [6, [13. Motivated by this fact, we point out also some particular phenomenons which occur in the case of pseudo-harmonic morphisms with one and two-dimensional fibers.
\end{abstract}

\section{INTRODUCTION}

Pseudo-harmonic morphisms are a special class of harmonic maps into a Hermitian manifold with the aditional property called Pseudo Horizontal Weak Conformality (PHWC), cf. 8, 10. This property generalises horizontal weak conformality, a geometrical condition satisfied by any harmonic morphism $\varphi$ and which translates into: $d \varphi \circ d \varphi^{*}=\lambda^{2} i d$, where $d \varphi^{*}$ stands for the adjoint map. Now, pseudo horizontal weak conformality of $\varphi: M \longrightarrow(N, J, h)$ will mean:

$$
\left[d \varphi \circ d \varphi^{*}, J\right]=0
$$

The geometric meaning of (1.1) will become more transparent if we remark that the differential of a submersion $\varphi$ from a Riemannian manifold $(M, g)$ into a Hermitian manifold $(N, J, h)$ induces an almost complex structure on the horizontal bundle, defined by $J_{\mathcal{H}}=\left.\left.d \varphi\right|_{\mathcal{H}} ^{-1} \circ J \circ d \varphi\right|_{\mathcal{H}}$. One can prove that PHWC condition is equivalent to the compatibility of $J_{\mathcal{H}}$ with domain metric $g$.

Moreover, if the codomain is Kähler, pseudo-harmonic morphisms have a nice description similar to harmonic morphisms. According to 10, pseudo-harmonic morphisms are those maps that pull back local complex-valued holomorphic functions on $N$ to local harmonic functions on $M$.

The analogue of horizontal homothety in this context was introduced by M.A. Aprodu, M. Aprodu and V. Brinzanescu in [3. A Pseudo-Horizontally Homothetic (PHH) map is a PHWC map $\varphi$ which satisfies:

$$
\left[d \varphi \circ \nabla_{X}^{M} \circ d \varphi^{*}, J\right]=0, \forall X \in \Gamma(\mathcal{H})
$$

In turn, this condition means that $J_{\mathcal{H}}$ is parallel (with respect to $\nabla^{\mathcal{H}}$ ) in horizontal directions, so satisfies a transversal Kähler condition. Any PHH harmonic submersion onto a Kähler manifold exhibits a particularly nice geometric property: it pulls back complex submanifolds into minimal submanifolds, cf. [3]. Moreover,

1991 Mathematics Subject Classification. 53C15, 58E20.

Key words and phrases. Riemannian manifolds, pseudo-harmonic morphisms, distribution. 
in [1] it is shown that PHH harmonic submersions are (weakly) stable (in particular such maps minimise the energy-functional in their homotopy class). Further properties and examples of $\mathrm{PHH}$ harmonic submersions can be found in [2], [5].

In this paper we shall place the study of pseudo-harmonic morphisms into the framework of transversely (almost) Hermitian distributions / foliations and also we shall explore some particular structure of pseudo-harmonic morphisms with one and two-dimensional fibers.

\section{Transversely almost Hermitian distributions}

In the begining we recall some basic notions in the study of distributions / foliations on a Riemannian manifold $\left(M^{m}, g\right)$. Let $\mathcal{V}$ and $\mathcal{H}$ be two orthogonal complementary distributions of dimension $m-n$ and $n$, respectively. Often, we shall call $\mathcal{V}$ and $\mathcal{H}$ the vertical and horizontal distributions. The exponent $\mathcal{V}$ or $\mathcal{H}$ will indicate the orthogonal projections onto these distributions. The second fundamental form $B^{\mathcal{H}}$ and the integrability tensor $I^{\mathcal{H}}$ of $\mathcal{H}$ are defined by:

$$
B^{\mathcal{H}}(X, Y)=\frac{1}{2}\left(\nabla_{X} Y+\nabla_{Y} X\right)^{\mathcal{V}}, \quad I^{\mathcal{H}}(X, Y)=[X, Y]^{\mathcal{V}}, \quad \forall X, Y \in \Gamma(\mathcal{H}) .
$$

Whether $\mathcal{H}$ is integrable or not, the mean curvature of $\mathcal{H}$ is, by definition:

$$
\mu^{\mathcal{H}}=\frac{1}{n} \operatorname{trace} B^{\mathcal{H}} .
$$

The Bott partial connection $\stackrel{\circ}{\nabla}=\stackrel{\circ}{\nabla}^{\mathcal{H}}$ on $\mathcal{H}$ is the map $\stackrel{\circ}{\nabla}: \Gamma(\mathcal{V}) \times \Gamma(\mathcal{H}) \longrightarrow \Gamma(\mathcal{H})$ defined by

$$
\stackrel{\circ}{\nabla}_{V} X=[V, X]^{\mathcal{H}} .
$$

Another connection which we work with is the normal connection, defined by:

$$
\nabla_{V}^{\mathcal{H}} X=\left(\nabla_{V} X\right)^{\mathcal{H}}, \forall V \in \Gamma(\mathcal{V}), X \in \Gamma(\mathcal{H}) .
$$

Definition 2.1. Let $\left(M^{m}, g\right)$ a Riemannian manifold. A distribution $\mathcal{V}$ of codimension $n$ will be called transversely (almost) Hermitian if the complementary distribution $\mathcal{H}$ admits an (almost) complex structure $J_{\mathcal{H}}$ compatible with the metric $g$. In particular, $n$ must be even.

Proposition 2.1. Let $\left(M^{m}, g\right)$ a Riemannian manifold equiped with a transversely almost Hermitian distribution $\mathcal{V}$. Then the following relations hold good:

(i.) $g\left(\left(\nabla_{E} J_{\mathcal{H}}\right)(X), X\right)=0, \forall E \in \Gamma(T M), X \in \Gamma(\mathcal{H})$

(ii.) $g\left(\left(\nabla_{E} J_{\mathcal{H}}\right)(X), J_{\mathcal{H}} X\right)=0, \forall E \in \Gamma(T M), X \in \Gamma(\mathcal{H})$

(iii.) $g\left(\left(\stackrel{\circ}{\nabla}_{V} J_{\mathcal{H}}\right)(X), X\right)=-\left(\mathcal{L}_{V} g\right)\left(X, J_{\mathcal{H}} X\right), \forall V \in \Gamma(\mathcal{V}), X \in \Gamma(\mathcal{H})$.

(iv.) $g\left(\left(\stackrel{\circ}{\nabla} V_{V} J_{\mathcal{H}}\right)(X), J_{\mathcal{H}} X\right)=-\frac{1}{2}\left(\mathcal{L}_{V} g\right)\left(J_{\mathcal{H}} X, J_{\mathcal{H}} X\right)+\frac{1}{2}\left(\mathcal{L}_{V} g\right)(X, X), \forall V \in$ $\Gamma(\mathcal{V})$,

$X \in \Gamma(\mathcal{H})$, where $\stackrel{\circ}{\nabla}$ denotes the Bott partial connection on $\mathcal{H}$.

Proof. The proof is essentially the same as for the Prop. 2.5 .16 (p.61) in reference [7.

Now let $\mathcal{V}$ be integrable so that it defines a foliation $\mathcal{F}$. If $\mathcal{V}$ is transversely almost Hermitian in the sense of the above definition, then we shall call $\mathcal{F}$ transversal almost Hermitian foliation if, in addition, the almost complex structure $J_{\mathcal{H}}$ is holonomy invariant (the metric is not necessarily holonomy invariant). 
The next result states that $J_{\mathcal{H}}$ induced by a PHWC submersion is holonomy invariant (i.e. $\stackrel{\circ}{\nabla}_{V} J_{\mathcal{H}}=0$ ). In particular, we obtain that a PHWC submersion always induces a transversely almost Hermitian foliation on the domain.

Lemma 2.1. Let $F^{\varphi}$ be the f-structure on $M$, naturally induced by a PHWC submersion $\varphi:(M, g) \longrightarrow(N, J, h)$ (F $F^{\varphi}$ extends $J_{\mathcal{H}}$ with zero on $\left.\mathcal{V}\right)$. Then, the following relation holds:

$$
\left(\left(\mathcal{L}_{V} F^{\varphi}\right)(X)\right)^{\mathcal{H}}=0, \quad \forall X \in \Gamma(\mathcal{H}) .
$$

Proof. By construction we have: $\left.d \varphi\right|_{\mathcal{H}} \circ J_{\mathcal{H}}=\left.J \circ d \varphi\right|_{\mathcal{H}}$. It is easy to see that if $X \in \Gamma(\mathcal{H})$ is basic (i.e. is also $\varphi$-related), then $J_{\mathcal{H}} X$ is basic too. Then it is trivial to check that: $d \varphi\left(\left(\mathcal{L}_{V} F^{\varphi}\right)(X)\right)=0$ is true for basic vector fields, so is true in general (we always have a basis of basic vector fields for the horizontal distribution of a submersion).

So we shall have as a direct consequence:

Corollary 2.1. In the above hypothesis we have the following equivalent relations:

(i) $\left(\mathcal{L}_{V} g\right)\left(J_{\mathcal{H}} X, J_{\mathcal{H}} X\right)=\left(\mathcal{L}_{V} g\right)(X, X), \forall V \in \Gamma(\mathcal{V}), X \in \Gamma(\mathcal{H})$.

(ii) $B^{\mathcal{H}}(X, X)=B^{\mathcal{H}}\left(J_{\mathcal{H}} X, J_{\mathcal{H}} X\right), \forall X \in \Gamma(\mathcal{H})$.

The following proposition is a characterization of transversal almost Hermitian foliations.

Proposition 2.2. A foliation $\mathcal{F}$ on a Riemannian manifold is transversely (almost) Hermitian if and only if for each $\mathcal{F}$-simple open set $U$, the leaf space $U / \mathcal{F}$ can be given a Hermitian structure with respect to which the natural projection $U \longrightarrow U / \mathcal{F}$ is a PHWC map.

Proof. The "only if" part has been already remarked, so we have to prove only the "if" part.

Let $U$ be a $\mathcal{F}$-simple open set in $M$. Denote $U / \mathcal{F}$ by $\bar{U}$ and by $\mathcal{V}$ the tangent distibution to $\mathcal{F}$. Then with the almost complex structure that we have on $\mathcal{H}$ one construct an almost complex structure on $\bar{U}$ in this way: $J=d \varphi \circ J_{\mathcal{H}} \circ d \varphi^{-1}$, where $\varphi$ denotes the standard projection and $d \varphi$ is restricted to $\mathcal{H}$. It is obvious that $J^{2}=-i d$ and that we can take a Riemannian metric $h$ on $N$, compatible with $J$. So we have got an almost Hermitian structure on $\bar{U}$.

Now we shall verify the PHWC condition in this context, that is:

$d \varphi \circ d \varphi^{*} \circ J=J \circ d \varphi \circ d \varphi^{*} \Leftrightarrow d \varphi^{*} \circ J \circ\left(d \varphi^{*}\right)^{-1}=d \varphi^{-1} \circ J \circ d \varphi \Leftrightarrow$ $d \varphi^{*} \circ J \circ\left(d \varphi^{*}\right)^{-1}=J_{\mathcal{H}}$.

In terms of asociated matrices, with obvious notations, this translates into:

$\left(G^{-1} \Phi^{t} H\right) J\left(H^{-1}\left(\Phi^{t}\right)^{-1} G\right)=J_{\mathcal{H}}$. But the compatibility of $J$ with the metric $h$ means simply: $H J=J H$, so the above relation becomes:

$\left(\Phi^{-1} J^{t} \Phi\right)^{t} G=G J_{\mathcal{H}} \Leftrightarrow J_{\mathcal{H}} G=G J_{\mathcal{H}}$, which is clearly satisfied by the hypothesis (compatibility of $J_{\mathcal{H}}$ with $g$ ).

\section{Transversely almost Hermitian foliations Which produce PSEudo HARMONIC MORPHISMS}

In this section we shall explore the conditions which a transversely almost Hermitian foliation must satisfy in order to be given locally by the fibers of a pseudo harmonic morphism. First we recall some results on harmonicity in this context. 
We know from [1] that for a PHWC map $\varphi:\left(M^{m}, g\right) \longrightarrow\left(N^{2 n}, J, h\right)$ from a Riemannian manifold to a Kähler one, the tension field of $\varphi$ is given by:

$$
\tau(\varphi)=-d \varphi\left(F^{\varphi} \delta F^{\varphi}\right),
$$

where $F^{\varphi}$ is the $f$-structure on $M$, naturally induced by the $\mathrm{PHWC}$ map $\varphi$ (in fact, $F^{\varphi}$ extends $J_{\mathcal{H}}$ with zero on $\mathcal{V}$ ) and $\delta F^{\varphi}=\operatorname{trace} \nabla F^{\varphi}$ is the divergence of $F^{\varphi}$.

If we consider an adapted frame $\left\{e_{i}, F^{\varphi} e_{i}, e_{\alpha}\right\}$ (i.e. an orthonormal frame such that $e_{\alpha} \in \operatorname{Ker} F^{\varphi}$ ), then the local form of the above formula is:

$$
\tau(\varphi)=-d \varphi\left(\sum_{i=1}^{n} F^{\varphi}\left[\left(\nabla_{e_{i}} F^{\varphi}\right)\left(e_{i}\right)+\left(\nabla_{F \varphi e_{i}} F^{\varphi}\right)\left(F^{\varphi} e_{i}\right)\right]+(m-2 n) \mu^{\mathcal{V}}\right) .
$$

Now, suppose that $\varphi$ is a pseudo-horizontally homothetic map. The condition (1.2) translates simply into $d \varphi\left(\left(\nabla_{X} F^{\varphi}\right) Y\right)=0, \forall X, Y \in \Gamma(\mathcal{H})$, so $\left[\left(\nabla_{X} F^{\varphi}\right) Y\right]^{\mathcal{H}}=$ $0, \forall X, Y \in \Gamma(\mathcal{H})$. This can be rewritten as follows:

$$
\left(\nabla_{X}^{\mathcal{H}} J_{\mathcal{H}}\right) Y=0, \forall X, Y \in \Gamma(\mathcal{H}) .
$$

In particular, $F^{\varphi} \operatorname{div}_{\mathcal{H}} F^{\varphi}=0$, so the formula (3.1) reduces to:

$$
\tau(\varphi)=-(m-2 n) d \varphi\left(\mu^{\mathcal{V}}\right) .
$$

So a pseudo-horizontally homothetic map is harmonic if and only if the fibers are minimal (see [3]).

By analogy with the case of harmonic morphisms (cf. [7]), we can introduce the following:

Definition 3.1. Let $(M, g)$ be a Riemannian manifold and $\mathcal{V}$ be a foliation on it. We say that $\mathcal{V}$ produces pseudo-harmonic morphisms on $(M, g)$ if each point of $M$ has an open neighbourhood $U$ which is the domain of a submersive pseudoharmonic morphism $\varphi:\left(U,\left.g\right|_{U}\right) \longrightarrow(N, J, h)$ whose fibers are open subsets of the leaves of $\mathcal{V}$.

Proposition 3.1. On a Riemannian manifold $\left(M^{m}, g\right)$, a transversely almost Hermitian foliation $\mathcal{V}$ of codimension $2 n$ produces pseudo-harmonic morphisms if and only if the following relation holds:

$$
2 n \mu^{\mathcal{H}}-(m-2 n) \mu^{\mathcal{V}}=\sum_{i=1}^{n}\left[\left(\nabla_{F e_{i}} F\right) e_{i}-\left(\nabla_{e_{i}} F\right) F e_{i}\right]
$$

where $F$ is the $f$-structure obtained by extension of $J_{\mathcal{H}}$ to $T M\left(\left.F\right|_{T \mathcal{H}}=J_{\mathcal{H}},\left.F\right|_{T \mathcal{V}}=\right.$ $0)$ and $\left\{e_{i}, F e_{i} \mid i=\overline{1, n}\right\}$ is an adapted frame in the complementary distribution $\mathcal{H}$.

Proof. The relation (3.1) tells us that a PHWC map $\varphi$ is harmonic if and only if

$$
\begin{aligned}
(m-2 n) \mu^{\mathcal{V}} & =-\sum_{i=1}^{n} F^{\varphi}\left[\left(\nabla_{e_{i}} F^{\varphi}\right)\left(e_{i}\right)+\left(\nabla_{F^{\varphi} e_{i}} F^{\varphi}\right)\left(F^{\varphi} e_{i}\right)\right] \\
& =-\sum_{i=1}^{n}\left[\nabla_{e_{i}}^{\mathcal{H}} e_{i}+\nabla_{F^{\varphi} e_{i}}^{\mathcal{H}} F^{\varphi} e_{i}+F^{\varphi}\left(\nabla_{e_{i}} F^{\varphi} e_{i}-\nabla_{F \varphi} e_{i} e_{i}\right)\right] .
\end{aligned}
$$

Combining this with $\mu^{\mathcal{H}}=1 / 2 n\left(\nabla_{e_{i}} e_{i}+\nabla_{F^{\varphi} e_{i}} F^{\varphi} e_{i}\right)^{\mathcal{V}}$ (cf. definition) and applying Prop.2.2, we obtain the stated result.

In the rest of this section, the notations are those one from [4. 
Corollary 3.1. On a contact metric manifold, the foliation associated to the Reeb vector field is obviously transversely almost Hermitian and it always produces pseudo harmonic morphisms.

If, in addition, the associated almost contact structure is normal (i.e. on a Sasakian manifold), the same foliation produces PHH pseudo harmonic morphisms.

Proof. Let $\left(M^{2 n+1}, \eta\right)$ be a contact manifold and $(\phi, \xi, \eta, g)$ an almost contact structure such that $d \eta(X, Y)=g(X, \phi Y)$.

For any $X$ and $Y$ we have Olszak's formula, cf. [12]:

$$
\left(\nabla_{X} \phi\right) Y+\left(\nabla_{\phi X} \phi\right) \phi Y=2 g(X, Y) \xi-\eta(Y)(X+h X+\eta(X) \xi) .
$$

If we put $Y=\phi X$, we obtain:

$$
\left(\nabla_{X} \phi\right) \phi X=\left(\nabla_{\phi X} \phi\right) X
$$

So, in this case, the right hand side of the formula (3.1) is zero, and our statement is now obviously true if we point out that, in this case, $\mathcal{H}=\mathcal{D}$ and $\mathcal{V}=S p\{\xi\}$ and therefore $\mu^{\mathcal{V}}=0$ (the integral curves of $\xi$ are even geodesics) and also $\mu^{\mathcal{H}}=0$ $(\mathcal{H}=\mathcal{D}$ being an $\phi$-invariant distribution so necessarily minimal).

The second statement will be a direct consequence of the Sasaki condition:

$$
\left(\nabla_{X} \phi\right) Y=g(X, Y) \xi-\eta(Y) X, \quad \text { for all } X, Y \in \mathcal{X}(M) \text {. }
$$

So, if we take $X, Y, Z \in \Gamma(\mathcal{D})$ and if we consider in both members only the component which is tangent to the contact distribution, then we obtain zero in the right hand side of (3.4). This means precisely $\nabla_{X}^{\mathcal{D}} \phi=0$, the PHH condition in this context.

It is worth to notice here that the foliation associated to the Reeb vector field on a contact metric manifold does not produce in general $\mathrm{PHH}$ pseudo harmonic morphisms, as it will be shown in the sequel. Nevertheless, $\phi$, which plays the role of the associated $f$-structure of the induced pseudo-harmonic morphism, will always be $(1,2)$-symplectic (i.e. $\left.\phi\left(\left(\nabla_{X} \phi\right) Y+\left(\nabla_{\phi X} \phi\right) \phi Y\right)=0, \forall X, Y \in \Gamma(\mathcal{D})\right)$.

Corollary 3.2. On a K-contact manifold which is not a Sasakian manifold, $\phi$ is a (1,2)-symplectic $f$-structure, but it is not parallel in horizontal directions (i.e. tangent to the contact distribution) whith respect to $\nabla^{\mathcal{D}}$.

Proof. The first assertion follows imediately from Olszak formula cited above. For the second, we shall use the following formula which holds good on any contact metric manifold (cf. 4, p.66):

$$
2 g\left(\left(\nabla_{X} \phi\right) Y, Z\right)=g\left(N^{(1)}(Y, Z), \phi X\right)+2 d \eta(\phi Y, X) \eta(Z)-2 d \eta(\phi Z, X) \eta(Y)
$$

. Suppose that $\nabla_{X}^{\mathcal{D}} \phi=0, \forall X \in \Gamma(\mathcal{D})$. Then, from the above formula, it follows for an arbitrary $X$ tangent to $\mathcal{D}$, that: $g\left(N^{(1)}(Y, Z), \phi X\right)=0, \forall Y, Z \in \Gamma(\mathcal{D})$. This implies that $\left(N^{(1)}(Y, Z)\right)^{\mathcal{D}}=0$, for any $Y, Z$ tangent to the contact distribution.

It is easy to see that the other component of $N^{(1)}(Y, Z)$, the one collinear with $\xi$, vanishes too: $g\left(N^{(1)}(Y, Z), \xi\right)=\eta([\phi X, \phi Y]-[X, Y]) \xi=0$ (this is because on a contact metric manifold, $\left.N^{(2)}=0\right)$.

Now, $N^{(1)}(Y, \xi)=\phi\left(\left(\mathcal{L}_{\xi} \phi\right) X\right)$ and because we are on a K-contact manifold, it must be zero too. We can conclude that $N^{(1)}=0$, which is clearly not the case if the manifold is not Sasakian. 
Examples of manifolds which are K-contact but not Sasakian could be found for instance in [14].

Remark 3.1. In particular, this proves that Prop.7 in [11] is indeed a generalisation of the corresponding result in [3] (which was not clear so far). Both results asserts that certain submersive pseudo harmonic morphisms onto a Kähler manifold pull back complex submanifolds into minimal submanifolds, the one in [11] under the $(1,2)$-symplectic hypothesis, the other $(\underline{3})$ under the PHH hypothesis (parallelism of the induced $f$-structure in horizontal directions).

\section{Pseudo-harmonic Morphisms With one-Dimensional Fibers}

Let $\varphi:\left(M^{2 n+1}, g\right) \longrightarrow\left(N^{2 n}, J, h\right)$ be a PHWC submersion with one dimensional fibers into a Kähler manifold. We have seen that the horizontal bundle $\mathcal{H}$ inherits an almost complex structure $J_{\mathcal{H}}$, so in particular is oriented. If $M$ is orientable, an assumption we adopt from now on, then the quotient bundle $\mathcal{V}=T M / \mathcal{H}$ is an orientable line bundle over $M$, hence trivial. Therefore it admits a globally defined nowhere vanishing section. Choosing a unitary section $\xi$ of this type, one obtain an almost contact metric structure $(\phi, \xi, \eta, g)$ on $M$. Indeed, if we define $\left.\phi\right|_{\mathcal{H}}=J_{\mathcal{H}}$ and $\left.\phi\right|_{\mathcal{V}}=0$ and also $\eta(X)=g(X, \xi), \forall X \in \Gamma(T M)$, then the all conditions in the definition of an almost contact metric structure are satisfied (i.e. $\phi^{2}=-I+\eta \otimes \xi$, $\eta(\xi)=0$ and $g(\phi X, \phi Y)=g(X, Y)-\eta(X) \eta(Y)$, cf. 44). Moreover $\varphi$ is then a $(\phi, J)$-holomorphic map (i.e. $d \varphi \circ \phi=J \circ d \varphi)$.

Some natural questions arise in this context. Because in this case $M$ is a foliated manifold (the foliation induced by the fibers, denoted by $\mathcal{F}_{\xi}$ ), one could ask which geometrical objects on $M$ are $\mathcal{F}_{\xi}$-foliated or $\mathcal{F}_{\xi}$-projectable? Under which additional hypothesis, $\phi$ is normal? Which geometrical aspects has the harmonicity in this context? Could be (partially) classified these particular harmonic maps as in the case treated in [6] and [13] We are going to answer here to some of these questions.

Lemma 4.1. Let $\varphi:\left(M^{2 n+1}, g\right) \longrightarrow\left(N^{2 n}, J, h\right)$ be a PHWC (surjective) submersion from an orientable Riemannian manifold into a Kähler manifold. With the above notations, we have the following:

(i) $\xi$ is Killing if and only if $\mathcal{H}$ is a totally geodesic distribution, invariant along the flow of $\xi$. In particular, in this case, $g$ is $\mathcal{F}_{\xi}$-foliated (or bundle-like for $\mathcal{F}_{\xi}$ ).

(ii) $[\phi, \phi](X, Y)=\eta([\phi X, \phi Y]) \xi$, for any horizontal vector fields $X, Y$ on $M$.

(iii) If in addition $\varphi$ is PHH submersion, then the fundamental 2-form

$\Phi(X, Y):=g(X, \phi Y)$ satifies: $d \Phi(X, Y, Z)=0, \forall X, Y, Z \in \Gamma(\mathcal{H})$.

Proof. (i) One can easily verify that:

$\left(\mathcal{L}_{\xi} g\right)(\xi, X)=\eta([X, \xi]), \quad \forall X \in \Gamma(\mathcal{H})$,

$\left(\mathcal{L}_{\xi} g\right)(X, Y)=-2 g\left(B^{\mathcal{H}}(X, Y), \xi\right), \quad \forall X, Y \in \Gamma(\mathcal{H})$.

Now the statement follows in a obvious way.

(ii) By definition, $\phi X=d \varphi^{-1} \circ J \circ d \varphi(X), \forall X \in \Gamma(\mathcal{H})$. We have noted that $d \varphi^{-1}$ stands for the horizontal lift application. For projectable horizontal vector fields $X, Y$ we shall have the following sequence of identities (similar to those one in 4, Example 6.7.2, p. 81): 


$$
\begin{aligned}
{[\phi, \phi](X, Y)=} & d \varphi^{-1} J^{2} d \varphi[X, Y]+\left[d \varphi^{-1} J d \varphi X, d \varphi^{-1} J d \varphi Y\right]- \\
& d \varphi^{-1} J d \varphi\left[d \varphi^{-1} J d \varphi X, Y\right]-d \varphi^{-1} J d \varphi\left[X, d \varphi^{-1} J d \varphi Y\right]= \\
& d \varphi^{-1} J^{2}[d \varphi X, d \varphi Y]+d \varphi^{-1}[J d \varphi X, J d \varphi Y]+ \\
& \eta\left(\left[d \varphi^{-1} J d \varphi X, d \varphi^{-1} J d \varphi Y\right]\right) \xi- \\
& d \varphi^{-1} J[J d \varphi X, d \varphi Y]-d \varphi^{-1} J[d \varphi X, J d \varphi Y]= \\
& d \varphi^{-1}[J, J][d \varphi X, d \varphi Y]+\eta\left(\left[d \varphi^{-1} J d \varphi X, d \varphi^{-1} J d \varphi Y\right]\right) \xi= \\
& \eta([\phi X, \phi Y]) \xi
\end{aligned}
$$

because $J$ is, by hypothesis, integrable.

(iii) We can check that:

$$
d \Phi(X, Y, Z)=g\left(X,\left(\nabla_{Z} \phi\right) Y\right)+g\left(Y,\left(\nabla_{X} \phi\right) Z\right)+g\left(Z,\left(\nabla_{Y} \phi\right) X\right) .
$$

The assertion follows imediately because PHH condition means exactly $\nabla_{X}^{\mathcal{H}} \phi=$ $0, \forall X \in \Gamma(\mathcal{H})$.

Note that the above argument for $(i i)$ can be used to show that for any PHWC submersion we have $\left[F^{\varphi}, F^{\varphi}\right](X, Y)=\left[F^{\varphi} X, F^{\varphi} Y\right]^{\mathcal{V}}, \forall X, Y \in \operatorname{Ker}\left(\left(F^{\varphi}\right)^{2}+I\right)$.

We recall now some standard facts on 1-dimensional foliations, pointed out in 7], section 10.5. In the following, the superscript $\mathcal{H}$ or $\mathcal{V}$ denotes the projection onto horizontal directions and fiber direction, respectively.

Remark 4.1. The 1-dimensional foliation $\mathcal{F}_{\xi}$ has minimal (i.e. geodesic) leaves (with respect to $g$ ) if and only if $\mathcal{L}_{\xi} \eta=0$ (which is equivalent to $\mathcal{L}_{\xi} \Gamma(\mathcal{H}) \subset \Gamma(\mathcal{H})$ as well as to $d \eta(\xi, X)=0$, for any horizontal field $X$ ).

The integrability tensor of the horizontal distribution is given by: $I^{\mathcal{H}}(E, F)=$ $\left[E^{\mathcal{H}}, F^{\mathcal{H}}\right]^{\mathcal{V}}, \forall E, F \in \Gamma(T M)$. Then $\Omega:=d \eta$ will be called integrability 2-form of $\mathcal{F}_{\xi}$. For $X, Y$ horizontal, we have:

$$
\Omega(X, Y)=-\eta([X, Y])=-g\left(I^{\mathcal{H}}(X, Y), \xi\right) .
$$

So $I^{\mathcal{H}}(X, Y)=-\Omega(X, Y) \xi$. In addition, for a foliation with minimal leaves, $\Omega$ is a basic form (i.e. $i_{\xi} \Omega=0$ and $\mathcal{L}_{\xi} \Omega=0$ ).

Note also that the natural decomposition of the metric: $g=g^{\mathcal{H}}+g^{\mathcal{V}}$ becomes in this case: $g=g^{\mathcal{H}}+\eta \otimes \eta$.

Theorem 4.1. Let $\varphi:\left(M^{2 n+1}, g\right) \longrightarrow\left(N^{2 n}, J, h\right)$ be a PHH harmonic submersion from an oriented Riemannian manifold into a Kähler manifold. Then:

(i) the almost contact structure naturally inherited by $M$ is normal if and only if $\nabla_{\xi} \phi=0$.

(ii) if, in addition the fibers are all connected of the same homotopy type, $\varphi$ is a (local) principal bundle.

Proof. ( $i$ ) In this case, $\phi$ asociates to a (local) vector field $X \in \Gamma(\mathcal{H})$ the vector field $\phi X=\widetilde{d \varphi}(J(d \varphi X))$, where $\widetilde{d \varphi}$ stands for the horizontal lift of the respective vector field. So it is clear that, in this context, $\mathcal{L}_{\xi} \phi=0$.

Acording to Lemma 4.1, we have that:

$$
[\phi, \phi](X, Y)^{\mathcal{H}}=0, \forall X, Y \in \Gamma(\mathcal{H}) .
$$

On the other hand, we have also: 
$[\phi, \phi](X, Y)^{\mathcal{V}}=\eta([\phi X, \phi Y]) \xi$, for horizontal fields $X, Y$

and

$[\phi, \phi](X, \xi)=\phi\left(\left(\mathcal{L}_{\xi} \phi\right) X\right)$.

We have already noted in Lemma 2.1 that $\mathcal{L}_{\xi} \phi=0$, so the normality of $\phi$ (i.e. $[\phi, \phi](X, Y)+2 d \eta(X, Y) \xi=0)$ in this case is equivalent to: $\eta([\phi X, \phi Y])=$ $\eta([X, Y]), \forall X, Y \in \Gamma(\mathcal{H})$, which in turn is equivalent to $N^{(2)}(X, Y)=0, \forall X, Y \in$ $\Gamma(\mathcal{H})$.

In order to finalise the proof, we shall apply [4,Lemma 6.1. In particular this lemma implies that:

$2 g\left(\left(\nabla_{\xi} \phi\right)(X), Y\right)=3 d \Phi(\xi, \phi X, \phi Y)-3 d \Phi(\xi, X, Y)+N^{(2)}(X, Y), \forall X, Y \in \Gamma(\mathcal{H})$, where $\Phi(X, Y):=g(X, \phi Y)$.

Now, precisely because $\mathcal{L}_{\xi} \phi=0$, we have $d \Phi(\xi, \phi X, \phi Y)-d \Phi(\xi, X, Y)=0$. Therefore $N^{(2)}(X, Y)=0, \forall X, Y \in \Gamma(\mathcal{H})$ is equivalent to $\left(\left(\nabla_{\xi} \phi\right) X\right)^{\mathcal{H}}=0$. It is easy to prove that under our hypothesis, $\left(\left(\nabla_{\xi} \phi\right) X\right)^{\mathcal{V}}=0$, for any $X$ horizontal (and obviously $\left.\left(\nabla_{\xi} \phi\right)(\xi)=0\right)$. This is because a PHH harmonic morphism has minimal fibers, cf. [3].

So a necessary and sufficient condition for the normality of $\phi$ will be $\nabla_{\xi} \phi=0$.

(ii) We know from [3] that a PHH submersion is harmonic if and only if it has minimal fibers. So, from the considerations above, $\mathcal{H}$ is invariant under the local flow of $\xi$. Therefore the integral curves of $\xi$ are the fibers of a local principal bundle with $\mathcal{H}$ a principal connection on it.

Note however that the PHH condition together with $\nabla_{\xi} \phi=0$ do not force $\phi$ to be parallel or equivalently, $(\phi, \xi, \eta, g)$ to be a cosymplectic structure in the sense of Blair (see 4], p. 77)

Remark 4.2. In the above hypothesis, condition $\nabla_{\xi} \phi=0$ is equivalent to

$$
d \eta(\phi X, \phi Y)=d \eta(X, Y), \quad \forall X, Y .
$$

Corollary 4.1. In the same hypothesis as above, if $M$ is compact and the vector field $\xi$ is regular, then $\varphi$ is a principal circle bundle.

\section{Pseudo-harmonic Morphisms With two-Dimensional Fibers}

First, we shall remark that any PHH harmonic map with 1-dimensional fibers from an orientable Riemannian manifold into a Kähler manifold gives rise to a naturally associated PHH harmonic map with 2-dimensional fibers.

We recall here that the cone $\mathcal{C}(M)$ over an almost contact metric manifold $\left(M^{2 n+1}, \phi, \xi, \eta, g\right)$ is simply $M^{2 n+1} \times \mathbb{R}$ with an almost complex structure defined by:

$$
J\left(X, f \frac{d}{d t}\right)=\left(\phi X-f \xi, \eta(X) \frac{d}{d t}\right) .
$$

We endow the cone with a warped product metric $\widehat{g}=d t \otimes d t+t^{2} g$. Let $\pi$ denote de standard projection $\pi: \mathcal{C}(M) \longrightarrow M$

Proposition 5.1. Let $\varphi:\left(M^{2 n+1}, g\right) \longrightarrow\left(N^{2 n}, J, h\right)$ be a smooth map and let $\widehat{\varphi}$ denote the map $\varphi \circ \pi$.

(i) If $\varphi$ is $(\phi, J)$-holomorphic, then $\widehat{\varphi}$ is holomorphic. The converse is true if and only if $\left(0, \frac{d}{d t}\right) \in \operatorname{Ker} d \widehat{\varphi}$ 
(ii) $\varphi$ is a PHH harmonic map if and only if so is $\widehat{\varphi}$.

Proof. (i) we have:

$$
d \widehat{\varphi}\left(J\left(X, f \frac{d}{d t}\right)\right)=d \varphi(\phi X-f \xi)=d \varphi(\phi X)=J d \varphi(X)=J\left(d \widehat{\varphi}\left(X, f \frac{d}{d t}\right)\right) .
$$

Conversely, if $\widehat{\varphi}$ is holomorphic then we have: $d \varphi(\phi X)-f d \varphi(\xi)=J d \varphi(X)$. But $d \varphi(\xi)=-J d \widehat{\varphi}\left(0, \frac{d}{d t}\right)$ and our assertion follows.

(ii) We have only to remark that $\pi$ is a horizontally homothetic harmonic morphisms. This is because it is obviously horizontally homothetic and we can easily check that its fibers are geodesics, so minimal.

So we are in the hypothesis of [3, Prop. 3.4, which states precisely that $\varphi$ is a $\mathrm{PHH}$ harmonic map if and only if so is $\widehat{\varphi}$.

Now, let $\varphi:\left(M^{2 n+2}, g\right) \longrightarrow\left(N^{2 n}, J, h\right)$ be a PHWC submersion with 2 - dimensional fibers, from an oriented Riemannian manifold into a Kähler manifold. Then, on $M$, we shall have two almost Hermitian structures with respect to which $\varphi$ is holomorphic (this will be called adapted almost Hermitian structure as in [7). Indeed, consider the natural split induced by the submersion $T M=\mathcal{H} \oplus \mathcal{V}$. On $\mathcal{H}$ we have the almost complex structure $J_{\mathcal{H}}$ (and therefore an orientation), compatible with the metric, induced by the PHWC condition. On $\mathcal{V}$, consider the orientation such that $\mathcal{H} \oplus \mathcal{V}$ has the orientation of $M$ and also let $J_{\mathcal{V}}$ be the almost Hermitian structure given by rotation through $+\pi / 2$. Then we have two adapted almost Hermitian structure on $M: J^{+}=\left(J_{\mathcal{H}}, J_{\mathcal{V}}\right)$ and $J^{-}=\left(J_{\mathcal{H}},-J_{\mathcal{V}}\right)$.

The first fact to be remarked is the following (which appear, in other context, also in [7]):

Remark 5.1. Under the above hypothesis, we have:

$$
\nabla_{E}^{\mathcal{V}} J_{\mathcal{V}}=0, \quad \forall E \in \Gamma(T M)
$$

where $\left(\nabla_{E}^{\mathcal{V}} J_{\mathcal{V}}\right)(V):=\left(\nabla_{E} J_{\mathcal{V}} V\right)^{\mathcal{V}}-J_{\mathcal{V}}\left(\nabla_{E} V\right)^{\mathcal{V}}, \forall V \in \Gamma(\mathcal{V})$

Proof. As in Proposition 2.1, we can prove that:

$g\left(\left(\nabla_{E} J_{\mathcal{V}}\right)(V), V\right)=g\left(\left(\nabla_{E} J_{\mathcal{V}}\right)(V), J_{\mathcal{V}} V\right)=0$.

Because $V$ and $J_{\mathcal{V}} V$ form in this case a basis in $\mathcal{V}$, the conclusion follows.

Now, we assert that Theorem 5.1 proved in 9] holds without the horizontally conformal hypothesis and we state it in our context. Recall that an almost complex submanifold $F$ of an almost Hermitian manifold $(M, J, g)$ is superminimal if $J$ is parallel along $F$ i.e. $\nabla_{V} J=0$ for all vector fields $V$ tangent to $F$.

Theorem 5.1. Let $\varphi:\left(M^{2 n+2}, g\right) \longrightarrow\left(N^{2 n}, J, h\right)$ be a PHWC submersion with complex 1-dimensional fibers, from an oriented Riemannian manifold into a Kähler manifold. If the fibers are superminimal with respect to (one of) the almost complex structures induced on $M$, then the respective almost complex structure must be integrable.

Proof. The proof is essentially the same as in 9]. We have only to prove that in our case the condition $\left[\mathcal{H}^{1,0}, \mathcal{H}^{1,0}\right]^{\mathcal{V}} \subset \mathcal{V}^{1,0}$ is also satisfied.

Acording to [15], Lemma 3.1, on the almost Hermitian manifold $\left(M, J^{+}, g\right)$, for any section $X$ of $\mathcal{H}$ and any vector $V$ tangent to $\mathcal{V}$, we have:

$$
g\left(\nabla_{J^{+} Z} X+\nabla_{Z} J^{+} X, V\right)=g\left(X,\left(\mathcal{L}_{V} J^{+}-\nabla_{V} J^{+}\right) Z\right), \quad \forall Z \in \Gamma(T M) .
$$


Now, applying Lemma 2.1 and the superminimality hypothesis, we obtain for $X, Y \in \Gamma(\mathcal{H}):$

$$
g\left(\nabla_{J^{+} Y} X+\nabla_{Y} J^{+} X, V\right)=0, \forall V \in \Gamma(\mathcal{V})
$$

. In particular we have also: $g\left(\left[X, J^{+} Y\right]+\left[J^{+} X, Y\right], V\right)=0$. From this we get imediately that $\left[\mathcal{H}^{1,0}, \mathcal{H}^{1,0}\right]^{\mathcal{V}}=0$.

Acknowledgements. I am grateful to my $\mathrm{PhD}$ advisor, professor $\mathrm{V}$. Brinzanescu, for his very rare and generous patience to pursuite me in this work and also for giving me many crucial hints.

\section{REFERENCES}

[1] M.A. Aprodu, PHH harmonic submersions are energy-minimizing, Preprint.

[2] M.A. Aprodu, M. Aprodu, Implicitly defined harmonic PHH submersions, Manuscripta Math. 100 (1999), 103-121.

[3] M.A. Aprodu, M. Aprodu, V. Brinzanescu, A class of harmonic submersions and minimal submanifolds, Int. J. Math. 11(9) (2000), 1177-1191.

[4] D. E. Blair, Riemannian Geometry of Contact and Symplectic Manifolds, Birkhauser Boston, Progress in Mathematics, vol.203 (2002).

[5] V. Brinzanescu, Pseudo-harmonic morphisms; applications and examples, The Fifth International Workshop on Differential Geometry and Its Application, Sept. 18-22, 2001, Timisoara, Romania, in Analele Univ.Timisoara, Vol. XXXIX (2001), 111-121.

[6] R. L. Bryant, Harmonic morphisms with fibers of dimension one, Commun. Anal. Geom. 8(2) (2000), 219-265.

[7] P. Baird, J. C. Wood, Harmonic Morphisms Between Riemannian Manifolds, Clarendon Press - Oxford (2003).

[8] J. Chen, Structures of certain harmonic maps into Kähler manifolds, Int. J. Math. 8 (1997), 573-581.

[9] S. Gudmundsson, J.C. Wood Harmonic Morphisms between Almost Hermitian Manifolds, Bollettino U.M.I. (7) 11-B (1997), Suppl. fasc. 2, 185-197.

[10] E. Loubeau, Pseudo harmonic morphisms, Int. J. of Math. 8 (1997), 943-957.

[11] E. Loubeau, X. Mo, Pseudo horizontally weakly conformal maps from Riemannian Manifolds into Kähler Manifolds, Beiträge zur Alg. und Geom. (to appear).

[12] Z. Olszak, On contact metric manifolds, Tohoku Math. J., 31 (1979), 247-253.

[13] R. Pantilie, Harmonic morphisms with one-dimensional fibers, Int. J. Math. 10(4) (1999), 457-501.

[14] Ph. Rukimbira, Rank and k-nullity of contact manifolds, Int. J. of Math. and Mathematical Sciences 20 (2004), 1025-1034.

[15] M. Svensson, Holomorphic foliations, harmonic morphisms and the Walczak formula, J. London Math. Soc.(2) 68(3) (2003), 781-794.

Faculty of Physics, Bucharest University, Romania

E-mail address: slobir@k.ro 